\title{
Manejo da obesidade: uma revisão narrativa dos tratamentos com foco na cirurgia
}

\section{metabólica}

\author{
Obesity management: a narrative review of treatments focusing on metabolic surgery \\ Manejo de la obesidade: una revisión narrativa de los tratamientos centrados en la cirugía \\ metabólica
}

Recebido: 25/01/2022 | Revisado: 02/02/2022 | Aceito: 10/02/2022 | Publicado: 16/02/2022

\author{
Gabrielly Senna Parussolo \\ ORCID: https://orcid.org/0000-0001-6800-9827 \\ UniversidadeFederal de Viçosa, Brasil \\ E-mail: gabrielly.parussolo@ufv.br \\ Beatriz Barakat \\ ORCID: https://orcid.org/0000-0001-8563-3832 \\ UniversidadeFederal de Viçosa, Brasil \\ E-mail: beatriz.barakat@ufv.br \\ Madalena Geralda Cupertino Ribeiro \\ ORCID: https://orcid.org/0000-0002-2658-6519 \\ UniversidadeFederal de Viçosa, Brasil \\ E-mail: madalena.cupertino@ufv.br \\ Lucas Inácio de Loyola Vinha \\ ORCID: https://orcid.org/0000-0001-7079-5329 \\ UniversidadeFederal de Viçosa, Brasil \\ E-mail: lucas.vinha@ufv.br \\ Bianca Ferreira de Santana \\ ORCID: https://orcid.org/0000-0002-2913-1966 \\ UniversidadeFederal de Viçosa, Brasil \\ E-mail: bianca.santana @ufv.br \\ Marlon Martins Moreira \\ ORCID: https://orcid.org/0000-0002-2890-7339 \\ UniversidadeFederal de Viçosa, Brasil \\ E-mail: marlon.moreira@ufv.br \\ Martha Elisa Ferreira de Almeida \\ ORCID: https://orcid.org/0000-0002-5906-2244 \\ Universidade Federal de Viçosa, Brasil \\ E-mail: martha.almeida@ufv.br
}

\begin{abstract}
Resumo
A obesidade é uma patologia que caracteriza como um uma doença crônica não transmissível que resulta do balanço energético positivo promovendo o acúmulo de tecido adiposo, e contribui para o desenvolvimento de inúmeras patologias. Alguns indivíduos com obesidade podem ter o tratamento pautado na realização de cirurgias metabólicas, quando a atividade física e a dietoterapia e/ou intervenção farmacológica não forem satisfatórias. Este artigo de revisão narrativa tem como objetivo descrever sobre o manejo da obesidade e seus tratamentos com foco na cirurgia metabólica. Para sua elaboração foram utilizados artigos, livros e seus capítulos, e outros materiais. Os procedimentos cirúrgicos metabólicos e as intervenções por técnicas alternativas para a perda de peso são divididas em técnicas de procedimento reversíveis e irreversíveis, realizados no estômago e/ou estômago e intestino delgado, que promovem efeitos colaterais e possíveis complicações pós-cirúrgicas, sendo que nos casos de disabsorção de nutrientes torna-se necessário a suplementação de algumas vitaminas e minerais. Conclui-se que as cirurgias metabólicas são alternativas eficazes para a perda de peso e no tratamento de outras comorbidades, entretanto podem gerar implicações póscirúrgicas e efeitos colaterais irreversíveis, sendo necessária a adoção de novos hábitos alimentares.
\end{abstract}

Palavras-chave: Obesidade; Suplementação; Trato digestório.

\begin{abstract}
Obesity is a pathology characterized as a chronic non-communicable disease that results from a positive energy balance promoting the accumulation of adipose tissue, and contributes to the development of numerous pathologies. Some individuals with obesity may have treatment based on metabolic surgery, when physical activity and diet therapy and/or pharmacological intervention are not satisfactory. This narrative review article aims to describe the management of obesity and its treatments with a focus on metabolic surgery. For its elaboration, articles, books and their chapters, and other materials were used. Metabolic surgical procedures and interventions using alternative techniques for weight loss are divided into reversible and irreversible procedure techniques, performed in the stomach
\end{abstract}


and/or stomach and small intestine, which promote side effects and possible post-surgical complications. In cases of nutrient malabsorption, supplementation of some vitamins and minerals is necessary. It is concluded that metabolic surgeries are effective alternatives for weight loss and in the treatment of other comorbidities, however they can generate post-surgical implications and irreversible side effects, requiring the adoption of new eating habits.

Keywords: Obesity; Supplementation; Digestive treatment.

\section{Resumen}

La obesidad es una patología caracterizada como una enfermedad crónica no transmisible que resulta de un balance energético positivo que promueve la acumulación de tejido adiposo y contribuye al desarrollo de numerosas patologías. Algunas personas con obesidad pueden tener un tratamiento basado en cirugía metabólica, cuando la actividad física y la dietoterapia y/o la intervención farmacológica no son satisfactorias. Este artículo de revisión narrativa tiene como objetivo describir el manejo de la obesidad y sus tratamientos con enfoque en la cirugía metabólica. Para su elaboración se utilizaron artículos, libros y sus capítulos, y otros materiales. Los procedimientos e intervenciones quirúrgicas metabólicas que utilizan técnicas alternativas para la pérdida de peso se dividen en técnicas de procedimiento reversibles e irreversibles, realizadas en el estómago y/o estómago e intestino delgado, que promueven efectos secundarios y posibles complicaciones posquirúrgicas.En casos de malabsorción de nutrientes, la suplementación de algunas vitaminas y minerales es necesario. Se concluye que las cirugías metabólicas son alternativas efectivas para la pérdida de peso y en el tratamiento de otras comorbilidades, sin embargo pueden generar implicaciones posquirúrgicas y efectos secundarios irreversibles, requiriendo la adopción de nuevos hábitos alimentarios.

Palabras clave: Obesidad; Suplementación; Tratamiento digestivo.

\section{Introdução}

A obesidade nas últimas décadas ganhou destaque mundial, caracterizando-se um evento de proporções globais (Brasil, 2021). No Brasil, o sobrepeso e a obesidade tornaram-se crescentes em ambos os sexos e faixas etárias, sendo mais expressiva naqueles que apresentam um menor rendimento familiar (Dias et al., 2017). Devido às suas complicações, tal patologia é considerada uma sindemia mundial, resultante do perfil alimentar inadequado, e do comportamento sedentário associado à inatividade física (Swinburn et al., 2019).

O excesso de peso e a obesidade são consequências provenientes da lipogênese, cujos ácidos graxos são armazenados no tecido adiposo (ex: visceral, subcutâneo e mesentérico), e são classificados mediante sua cor e função (ex: branco, marrom e bege) (Zieba et al., 2019; Barakat \& Almeida, 2021). Tal condição apresenta-se um importante fator de risco para várias doenças crônicas não transmissíveis (DCNT) como as doenças cardiovasculares, diabetes mellitus, hipertensão arterial, doença hepática gordurosa não alcóolica, alguns tipos de cânceres (ex: mama, reto e cólon), e a cirrose (Martínez, 2017; Brasil, 2019).

A obesidade é uma patologia grave com elevados gastos para a saúde pública. De acordo a pesquisa realizada em 2019 pela Vigilância de Fatores de Risco e Proteção para Doenças Crônicas por Inquérito Telefônico (VIGITEL), a prevalência de excesso de peso no Brasil foi de 55,4\%, sendo 57,1\% nos homens e 53,9\% nas mulheres, enquanto 20,3\% de ambos os sexos apresentaram obesidade (Brasil, 2019).

O diagnóstico nutricional de obesidade é feito a partir de métodos antropométricos e/ou de equipamentos com intuito de verificar a composição corporal (Brasil, 2011). Os dados coletados na avaliação do paciente auxiliarão no diagnóstico nutricional a partir do Índice de Massa Corporal (IMC) para identificar o excesso de peso corporal; a razão circunferência da cintura/quadril que é um indicador com potencial significativo para o diagnóstico de doenças crônicas não transmissíveis; e as dobras cutâneas auxiliando na definição da porcentagem de gordura corporal. A bioimpedância elétrica, ressonância magnética, ultrassonografia, tomografia computadorizada, e a densitometria com a medida da gordura (DEXA) contribuem com o diagnóstico (Araújo et al., 2019; Oliveira et al., 2020).

$\mathrm{O}$ tratamento empregado a esta patologia deve ser feito por uma abordagem multiprofissional e transdisciplinar, com base na dietoterapia conciliada à atividade física, na utilização de fármacos antiobesidade para reduzir o apetite e o peso corporal, e nas cirurgias metabólicas (Andrade et al., 2019). As cirurgias metabólicas e os procedimentos endoscópicos apesar de promoverem resultados rápidos, são alternativas reversíveis ou irreversíveis que necessitam da compreensão do paciente 
para um novo estilo de vida com hábitos alimentares saudáveis (Florido et al., 2019). Tais cirurgias podem promover complicações pós-cirúrgicas como a deiscência da sutura, estenose, fístulas, infecções e hemorragia, hérnia interna e obstrução intestinal e/ou tromboembolismo pulmonar (Associação Brasileira para o Estudo da Obesidade e da Síndrome Metabólica ABESO, 2016), além de náuseas, diarreia, deficiências de algumas vitaminas e minerais, flatulência, e doenças osteometabólicas. As suplementações pós-cirúrgicas em cirurgias disabsortivas são uma demanda necessária, sendo este um dos pontos negativos das cirurgias metabólicas (Marra et al., 2021).

O objetivo deste artigo é descrever sobre o manejo da obesidade através de uma revisão narrativa dos tratamentos com foco na cirurgia metabólica.

\section{Metodologia}

Este estudo é uma revisão narrativa (Marques \& Quintilio, 2021), na qual se utilizaram-se as seguintes etapas:

- Etapa 1 - elaboração da pergunta norteadora: Como a cirurgia metabólica pode contribuir no tratamento da obesidade?

- Etapa 2 - definição dos descritores.

- Etapa 3 - identificação dos materiais. Foram identificados materiais como artigos, livros e capítulos de livros, e outros materiais publicados nos últimos 5 anos (2017 até 2021). As pesquisas foram extraídas das plataformas Scielo, Pubmed e Google Acadêmico, utilizando os descritores em português (Bypass Gástrico, Cirurgia metabólica, banda gástrica, sleeve, obesidade, compostos bioativos, fármacos, dietoterapia e complicações cirúrgicas), em inglês (Gastric bypass, metabolic surgery, gastric band, sleeve, obesity, bioactive compounds, drugs, diet therapy and surgical complications) e em espanhol (Bypass gástrico, cirugía metabólica, banda gástrica, manga, obesidad, compuestos bioactivos, fármacos, dietoterapia y complicaciones quirúrgicas).

- Etapa 4 - criação dos critérios de elegibilidade. Houve a comparação dos títulos dos artigos selecionados para excluir aqueles que apresentavam publicação duplicada nas bases consultadas. Após a exclusão dos artigos duplicados, seus títulos e resumos foram lidos para a identificação de sua elegibilidade, sendo que em seus títulos deveriam possuir pelo menos uma das palavras-chave utilizadas nas buscas ou abordar assuntos de interesse nesta pesquisa;

- Etapa 5 - análise e inclusão dos estudos. Ocorreu a seleção dos materiais com a leitura na íntegra de todos os artigos selecionados para a exclusão daqueles que não apresentavam informações relevantes ao estudo. Na elaboração do artigo foram utilizados 53 artigos, 3 livros, e 6 demais materiais;

- Etapa 6 - inclusão e leitura detalhada das 59 referências que abordavam diretamente o tema;

- Etapa 7 - elaboração da figura 1 na plataforma online Biorender;

- Etapa 8 - redação do artigo científico.

\section{Resultados e Discussão}

Os profissionais nutricionistas, psicólogos, educadores físicos, e médicos são importantes para o tratamento da obesidade, pois são responsáveis pelo restabelecimento do seu estado nutricional (Brasil, 2019; Castilho et al., 2021). A intervenção pode ser feita através da dietoterapia, atividade física, uso de medicamentos e/ou cirurgias metabólicas, obtendo uma conduta individualizada de acordo com a necessidade do paciente (Barroso et al., 2017). Mediante o fato da obesidade se associar aos distúrbios psíquicos, o acompanhamento de psicólogos contribuirá positivamente na sua evolução (Castilho et al., 2021). 


\subsection{Obesidade e dietoterapia}

Os hábitos alimentares são oriundos de fatores como as condições socioeconômicas, culturais, memórias afetivas, e a palatabilidade. Todas essas características devem ser levadas em consideração para a formulação e aplicação dietoterápica, a fim de reduzir o tecido adiposo, alterar sua composição corporal, e promover saúde (Santos et al., 2020).

Uma estratégia para o controle da obesidade é através do balanço energético negativo, onde o indivíduo precisa apresentar um consumo energético inferior ao seu Gasto Energético Total (GET) e superior ou igual sua Taxa Metabólica Basal (TMB) para atender suas necessidades mínimas de macro e micronutrientes (Barroso et al., 2017; Florido et al., 2019). A distribuição de macronutrientes é importante, visto que a obesidade se caracteriza pelo acúmulo de gordura visceral e subcutânea. Uma dieta rica em carboidratos e/ou lipídios contribui com o desenvolvimento ou agravamento das doenças cardiovasculares, pois diminui a lipoproteína de alta densidade (HDL), e aumenta a lipoproteína de baixa densidade (LDL), o colesterol total e os triacilgliceróis (Barroso et al., 2017; Florido et al., 2019).

Os macronutrientes devem apresentar concentrações adequadas e individualizadas, pois contribuem diretamente com as calorias ingeridas (De La Iglesia et al., 2016). Os carboidratos atuam como fontes primárias de energia, sendo a glicose a principal molécula usada pelo cérebro, e sua contribuição no plano alimentar faz-se necessária para a produção energética do indivíduo (De La Iglesia et al., 2016). As proteínas contribuem com a síntese de novas moléculas proteicas com destaque para a constituição dos tecidos musculares, enquanto os lipídios contribuem principalmente com a reserva energética e o isolamento térmico. O consumo excessivo de moléculas energéticas em dietas hiperglicídicas e/ou hiperlipídicas contribui para o desenvolvimento da obesidade, justificando a necessidade de dietas individualizadas, equilibradas e saudáveis (Brasil, 2017).

A biodisponibilidade alimentar caracteriza-se pelo aproveitamento do nutriente ingerido, e deve ser observado com cuidado na elaboração do plano alimentar. Devem ser utilizadas proteínas de alto valor biológico, que possuem altas concentrações dos aminoácidos essenciais, encontradas principalmente nos alimentos de origem animal (carne, leite e ovos). Este nutriente terá um importante papel estrutural na composição corpórea, visto que os aminoácidos essenciais não são provenientes da síntese humana, e devem ser ingeridos diariamente (De La Iglesia et al., 2016).

As fibras dietéticas mais e menos solúveis (Gallagher, 2012) apresentam destaque devido seu auxílio na formação do bolo fecal, prevenção de câncer de cólon, e redução da absorção lipídica que auxilia na obesidade e suas comorbidades como o diabetes mellitus, pois atuam retardando a absorção de glicose (Carvalho et al., 2020).

Os compostos bioativos são importantes para a prevenção e o controle da obesidade, que é uma patologia inflamatória crônica. As moléculas de carotenoides, catequinas, fitoesteróis, flavonoides, polifenóis, e resveratrol apresentam características anti-inflamatórias que promovem a modulação da inflamação no processo de obesidade, além de auxiliarem na formação do bolo fecal, e na alteração no tempo de absorção de macronutrientes (Braga et al., 2021; Silva; Santos; Almeida, 2021).

Os pré-bióticos, pró-bióticos e simbióticos são importantes para a saúde e auxiliam na síntese de moléculas antiinflamatórias, estimulam o crescimento de células intestinais, e contribuem para o fortalecimento do sistema imunológico ativando as células TCD4+ que exercem um papel importante na disbiose promovida pela obesidade (Cuevas-Sierra et al., 2019).

\subsection{Obesidade e atividade física}

A prática regular de atividade física é recomendada, entretanto sua conduta demanda o acompanhamento de um profissional qualificado, para evitar lesões ou impactos negativos para a saúde do indivíduo. É necessário que o indivíduo seja submetido a uma avaliação de resistência física e de patologias, para determinar os planos alimentares e de exercícios individualizados (Murai, 2021; Rossini et al., 2021). A atividade física contribui para a alteração da composição corporal e promove a aptidão física, melhora o condicionamento cardiovascular pela maior capacidade corporal de transportar e utilizar 
oxigênio. Pode ser feita de forma anaeróbica, aeróbica ou exercícios de força muscular, sendo que ambos promovem a lipólise e o aumento da termogênese (Martínez et al., 2017; Rossini et al., 2021).

O balanço energético negativo, proveniente da alimentação e o gasto energético do indivíduo pode ser afetado pela prática de atividade física, pois o exercício físico necessita de uma demanda energética, e contribui na promoção e desenvolvimento de massa muscular magra e na diminuição do percentual de gordura corporal (Christinelli et al., 2020). Os exercícios de força induzem a síntese da massa magra, enquanto o aeróbico demanda um gasto energético com o aumento de VO2 max que é eficaz para o processo do emagrecimento (Rossini et al., 2021).

\subsection{Obesidade e psicologia}

A obesidade sofre influência de diversos parâmetros fisiológicos, biológicos, e psicossociais que devem ser tratados com cautela para a obtenção de resultados positivos (Lubans et al., 2016). As mídias sociais interferem diretamente no desenvolvimento de patologias psíquicas, pois a busca do "corpo perfeito" tem sido um dos assuntos mais relevantes, e afeta negativamente a população (Rigoni; Nunes; Fonseca, 2017).

A saúde mental influencia na qualidade de vida e no consumo alimentar, e pode causar depressão, ansiedade, e transtornos alimentares que impactam na quantidade dietética ingerida, pois os indivíduos geralmente consomem alimentos em excesso ou insuficientes para sua demanda energética, sendo que ambos os casos são prejudiciais à saúde (Rigoni, 2017; Novelle; Diéguez, 2018).

Os distúrbios psíquicos podem propiciar uma inadequação no consumo alimentar, onde o consumo elevado de alimentos ultraprocessados com alto teor de carboidratos e/ou lipídios, e com uma grande palatabilidade pode ser um aliado negativo quando conciliado ao distúrbio alimentar, pois contribuem para o elevado consumo que contribui com a obesidade (Lubans et al., 2016; Novelle; Diéguez, 2018).

A influência de um profissional faz-se necessária para que seja possível o diagnóstico dos transtornos psíquicos, e para auxiliar nas situações de crise ou estresse, além de estabelecer uma relação mais adequada do paciente para a alimentação (Rigoni et al., 2017).

\subsection{Obesidade e farmacologia}

Os fármacos antiobesidade possuem a finalidade de diminuir o apetite, inibir a absorção de lipídios ou aumentar o gasto energético e intensificar a termogênese, com ação em neurotransmissores que promovem a recaptação de serotonina (Almeida; Uhlmann, 2021). Sua utilização deve ocorrer de forma secundária, quando os indivíduos não conseguem atingir a perda de peso necessária com a inserção de mudanças no estilo de vida, ou quando desenvolvem patologias decorrentes da obesidade que possam oferecer algum risco à sua saúde (Associação Brasileira para o Estudo da Obesidade e da Síndrome Metabólica - ABESO, 2019).

Os medicamentos anorexígenos, conhecidos de acordo a inibidores de apetite, ainda enfrentam problemas com sua regulamentação, sendo que na RDC no 52 de 2011 a Agência Nacional de Vigilância Sanitária (ANVISA) os retirou do mercado por não apresentarem eficácia e segurança de acordo os padrões farmacológicos, o que propiciou sua venda ilegal (Andrade et al., 2019). Nos Estados Unidos da América (EUA), a Agência Regulatória Food And Drug Administration (FDA) regulamentou na terapia antiobesidade os fármacos: Orlistat (Xenical - Inibidor da lipase gastrointestinal; Antiobesidade; Redutor de gordura), Lorcaserina (Belviq - Anorexígeno, Antiobesidade), Fentermina/Topiramato (Qsymia - Derivado anfetamínico/anticonvulsivante), Bupropiona/Naltrexona (Contrave - Antidepressivo heterocíclico de $2^{\text {a }}$ geração/Antagonista opioide), e a Liraglutida (Saxenda - Agonista do receptor peptídeo - 1 semelhante ao glucagon (GLP-1) (Almeida et al., 2019; Andrade, 2019). 
No Brasil, em 2011 a Agência Nacional de Vigilância Sanitária (ANVISA) retirou do mercado três substâncias inibidoras de apetite do tipo anfetamínicos (ex: Anfepramona, Femproporex e Mazindol), pois os Laboratórios que tinham registros desses medicamentos não apresentaram estudos de eficácia e segurança dentro dos padrões estabelecidos (ANVISA, 2018). Os fármacos inibidores de apetite são divididos em: catecolaminérgicos (ex: Anfepramona, Femproporex e Mazindol); serotoninérgicos (ex: Fluoxetina e Sertralina); catecolaminérgicos e serotoninérgicos (ex: Sibutramina); termogênicos (ex: Cafeína e Efedrina); inibidores da absorção de gorduras (ex: Orlistate); e antagonista seletivo de CB - 1 (ex: Rimonabanto) (Marques et al., 2021).

\subsection{Obesidade e cirurgias metabólicas}

Em 1954, após estudos experimentais os médicos Kremen e Liner realizaram a primeira cirurgia metabólica para a perda de peso, utilizando a técnica disabsortiva de desvio intestinal (Bypass), na qual se retirou 90\% do jejuno e do íleo (Costa et al., 2020). Em 1960, o médico americano Edward Mason aprimorou o Bypass, surgindo então o Bypass gástrico com grampos cirúrgicos para diminuir o estômago. Em 1980 os processos cirúrgicos para redução de peso passaram a ser reconhecidos como técnicas de eficácia com o apoio do National Institute of Healt (NIH), da Society of American Gastrointestinal and Endoscopic Surgeons (SAGES) e da American Gastrointestinal Association (AGA) (Neis et al., , 2018; Costa et al., 2020).

No Brasil, em 1970 Salomão Chaib não obteve sucesso ao realizar a primeira cirurgia metabólica para a redução de peso, no Hospital das Clínicas em São Paulo, utilizando a técnica de derivação intestinal (Costa et al., 2020). Ao longo dos anos muitos estudos e cirurgias metabólicas foram realizadas, e em 2001 ocorreu o primeiro procedimento pelo Sistema Único de Saúde (SUS) (Associação Brasileira para o Estudo da Obesidade e da Síndrome Metabólica - ABESO, 2009). Segundo a Lei no 6.343/2019, os indivíduos que possuem diabetes mellitus tipo 2 e não conseguem controlar através de hipoglicemiantes orais e alimentação podem realizar a cirurgia metabólica no programa de rede pública do Distrito Federal (Sociedade Brasileira de Cirurgia Bariátrica e Metabólica - SBCBM, 2019), sendo que o Hospital Regional da Asa Norte (HRAN) realizou em 2019 a primeira cirurgia metabólica para o tratamento de diabetes mellitus tipo 2 (Fortes et al., 2021).

Os procedimentos indicados para os pacientes obesos e com diabetes mellitus tipo 2 são a técnica de derivação gastrojejunal em y de roux ou a gastrectomia vertical, e deve ser feita necessariamente por dois médicos especialistas em endocrinologia (Brasil, 2017).

As cirurgias metabólicas são utilizadas para solucionar a problemática da obesidade, quando o paciente já submeteu aos processos dietoterápicos e/ou a prática de atividade física para promoção do emagrecimento (Lima et al., 2020; Braga et al., 2021). Ao ser submetido a uma cirurgia metabólica informações precisam ser previamente esclarecidas quanto as suas características irreversíveis ou reversíveis, disabsortivas e/ou que necessitam de suplementação pós-cirúrgica, além dos efeitos colaterais como enjoos, refluxo e/ou diarreia (Chansaenroj et al., 2017; Zilberstein; Santos; Carvalho, 2019).

Para ser apto a realização de cirurgias metabólicas, o paciente deve-se possuir os parâmetros presentes na Resolução no 2.131 de 2015 como a idade maior que 18 anos, IMC $>35 \mathrm{Kg} / \mathrm{m}^{2}$ ou IMC abaixo $35 \mathrm{Kg} / \mathrm{m}^{2}$ associado à comorbidades (diabetes mellitus, apneia do sono, hipertensão arterial, dislipidemia, doenças cardiovasculares incluindo doença arterial coronariana, infarto do miocárdio angina, insuficiência cardíaca congestiva, acidente vascular cerebral, hipertensão e fibrilação atrial, cardiomiopatia dilatada, cor pulmonale e síndrome de hipoventilação, asma grave não controlada, osteoartroses, hérnias discais, refluxo gastroesofageano com indicação cirúrgica, colecistopatia calculosa, pancreatites agudas de repetição, esteatose hepática, incontinência urinária de esforço da mulher, infertilidade masculina e feminina, disfunção erétil, síndrome dos ovários policísticos, veias varicosas e doença hemorroidária, hipertensão intracraniana idiopática, estigmatização social e depressão), e não fazer uso de álcool, drogas e/ou tabagismo (Brasil, 2019). 
A cirurgia bariátrica possui potencial de diminuição de até $48 \%$ o risco de mortalidade por COVID-19, diminuição de $74 \%$ o risco de UTI, e $64 \%$ de intubação, entretanto o procedimento cirúrgico não contribui diretamente com a imunização, mas diminui os riscos acometidos pelo SARS-Cov-2 (Sociedade Brasileira de Cirurgia Bariátrica e Metabólica - SBCBM, 2021).

As cirurgias metabólicas podem ser realizadas no estômago e/ou intestino delgado. Quando ocorre apenas no estômago tem-se a redução do espaço para promover saciedade precoce, logo se obtém como consequência o emagrecimento. As cirurgias mistas impactam na absorção de nutrientes no intestino delgado o que promove o emagrecimento de forma mais rápida (Marra et al., 2021). Dentre os procedimentos aceitos têm-se as técnicas restritivas [balão intragástrico; gastroplastia vertical (ex: cirurgia de mason), e banda gástrica ajustável]; disabsortivas (ex: Bypass Jejuno- jejunal - Payne), e mistas (ex: Bypass gástrico em y de Roux - Cirugia de Fobi-Capella ou Gastroplasia em Y de Roux) (Brasil, 2017; Brasil, 2020).

Dentre as cirurgias metabólicas realizadas no estômago (Figura 1), cita-se o balão intragástrico que é um procedimento alternativo que consiste na introdução de um balão inflável que promove a saciedade e a plenitude gástrica, e reduz o peso corporal (Zilberstein; Santos, Carvalho, 2019; Costa et al., 2020).

Figura 1: Cirurgias metabólicas ocorridas no estômago e/ou no intestino delgado.

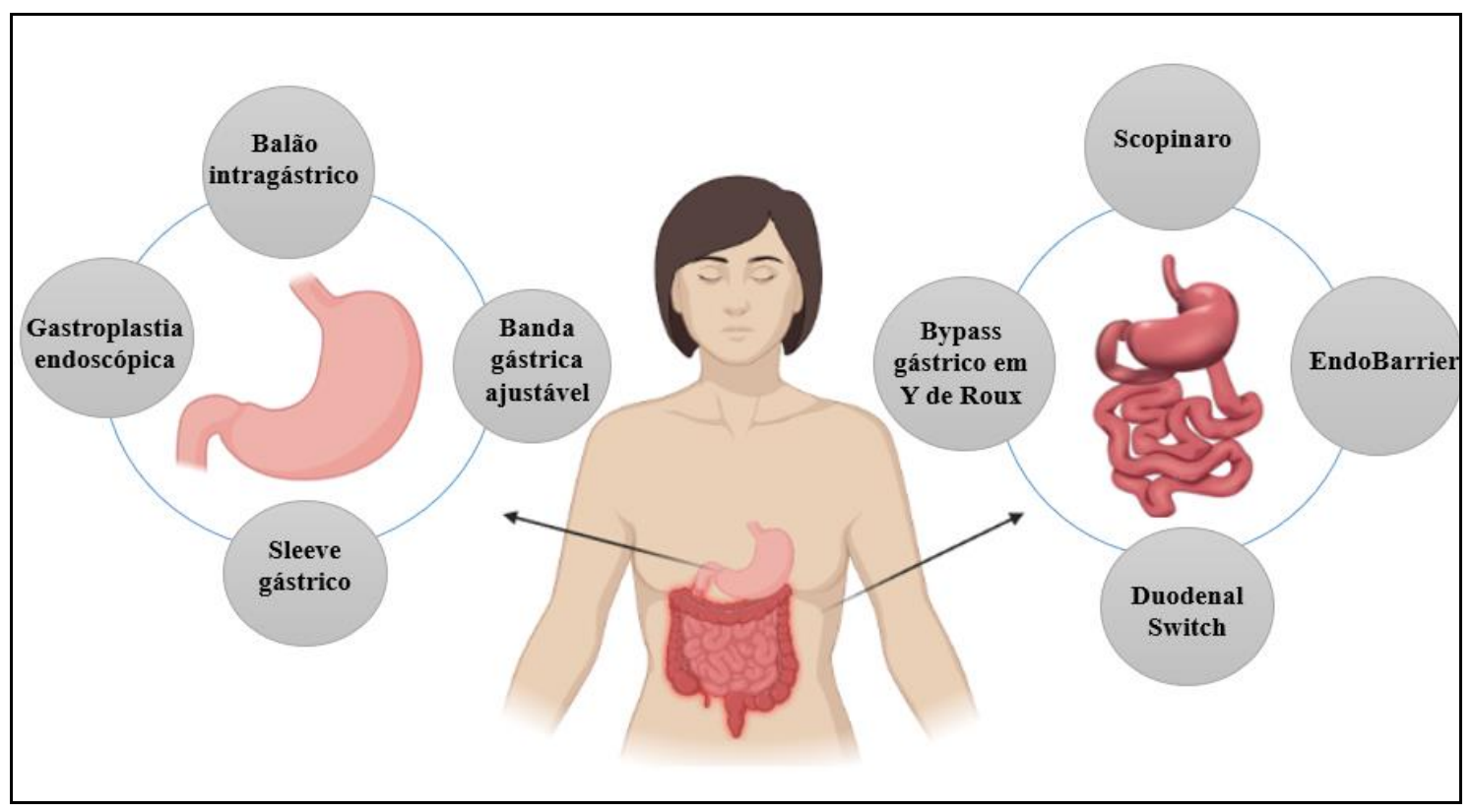

Fonte: Autores (2021).

A banda gástrica ajustável realizada no estômago proximal é uma cirurgia restritiva realizada por vídeo-laparoscopia. Esse procedimento é oriundo de uma banda que dificulta a passagem do alimento (Chansaenroj et al., 2017).

O sleeve gástrico (gastrectomia em manga, gastrectomia longitudinal, gastrectomia sleeve, gastrectomia vertical) é um procedimento para remover de 70 a 80\% do estômago proximal ao antro para reduzir a produção do hormônio grelina (Brasil, 2010). A retirada de parte do estômago através de grampeamento possui a finalidade de diminuir a capacidade do estômago, sem a necessidade de desvio intestinal, e apresenta características restritivas e disabsortiva (Chung et al., 2018). É indicado para pacientes com IMC $\geq 40 \mathrm{~kg} / \mathrm{m}^{2}$ ou com IMC $=35 \mathrm{~kg} / \mathrm{m}^{2}$ associado à comorbidades como diabetes mellitus, apneia do sono, hipertensão arterial, dislipidemia, doença arterial coronariana, infarto do miocárdio angina, insuficiência cardíaca congestiva, acidente vascular cerebral, hipertensão e fibrilação atrial, cardiomiopatia dilatada, cor pulmonale e síndrome da hipoventilação, asma grave não controlada, osteoartroses, hérnias discais, doença do refluxo gástrico-esofágico com indicação cirúrgica, 
colecistopatia calculosa, pancreatites agudas de repetição, esteatose hepática, incontinência urinária de esforço na mulher, infertilidade masculina e feminina, disfunção erétil, veias varicosas e doença hemorroidária, hipertensão intracraniana idiopática, estigmatização social e depressão (Brasil, 2015).

A gastroplastia endoscópica é um método alternativo sem cortes, realizado no estômago para reduzir sua capacidade por grampeamento, sendo um método reversível e rápido (Glass et al., 2019).

As cirurgias metabólicas do estômago apresentam várias vantagens e desvantagens, conforme pode ser visto no Quadro 1:

Quadro 1: Vantagens e desvantagens das cirurgias metabólicas no estômago.

\begin{tabular}{|c|c|c|}
\hline Tipo de cirurgia & Vantagens & Desvantagens \\
\hline Balão intragástrico & $\begin{array}{l}\text { - Reversível; } \\
\text { - Menos invasiva; } \\
\text { - Opção alternativa para obesidade; } \\
\text { - Indolor. }\end{array}$ & $\begin{array}{l}\text { - Promove náuseas e vômitos; } \\
\text { - Perda de peso pouco considerável (média } 15 \mathrm{~kg} \text { ); } \\
\text { - Possibilidade de reganho após sua retirada. }\end{array}$ \\
\hline Banda gástrica ajustável & $\begin{array}{l}\text { - Reversível; } \\
\text { - Ajuste de restrição; } \\
\text { - Pouco agressiva; } \\
\text { - Procedimento simples. }\end{array}$ & $\begin{array}{l}\text { - Tende a contribuir com um hábito alimentar hipercalórico; } \\
\text { - Promoção de refluxo gastroesofágico; } \\
\text { - Material sintético (prótese de silicone) que pode promover } \\
\text { a repulsão fisiológica do material; } \\
\text { - Perda de peso pode ser insuficiente. }\end{array}$ \\
\hline Sleeve gástrico & $\begin{array}{l}\text { - Perda de peso significativa; } \\
\text { - Não provoca a Síndrome de } \\
\text { Dumping; } \\
\text { - Diminui a produção de grelina. }\end{array}$ & $\begin{array}{l}\text { - Irreversível; } \\
\text { - Promoção de refluxo gastroesofágico; } \\
\text { - Possíveis desenvolvimentos de patologias nutricionais; } \\
\text { - Necessário a suplementação (entretanto sua recomendação } \\
\text { é usada em um menor período quando comparada ao Bypass } \\
\text { em y de roux). }\end{array}$ \\
\hline $\begin{array}{l}\text { Gastroplastia endoscópica ou } \\
\text { Gastroplastia endoscópica } \\
\text { vertical }\end{array}$ & $\begin{array}{l}\text { - Rápido; } \\
\text { - Minimamente invasivo; } \\
\text { - Recuperação rápida; } \\
\text { - Reversível. }\end{array}$ & $\begin{array}{l}\text { - Tende ao insucesso; } \\
\text { - Contribui para reganho de peso; } \\
\text { - Ao longo do tempo o grampeamento pode se soltar. }\end{array}$ \\
\hline
\end{tabular}

Fonte: Autores (2021).

Scopinaro é uma cirurgia disabsortiva com derivação bilipancreática pouco realizada, que envolve o estômago, intestino delgado (ex; dudodeno, jejuno e íleo), e o intestino grosso. Inicialmente ocorre a secção do estômago, e posteriormente é realizada a anastomose do intestino até o estômago pequeno, diminuindo o percurso do alimento de $7 \mathrm{~m}$ para 2,5 m (Costa et al., 2020).

O EndoBarrier é um tubo de silicone que é inserido por videolaparoscopia no duodeno. É menos agressivo e reversível, com a vantagem da alta hospitalar no mesmo dia do procedimento (Ruban et al., 2018).

Duodenal Switch ou cirurgia disabsortiva com derivação bilipancreática ocorre pela redução do estômago por gastrectomia vertical. Para ocorrer a anastomose é feita a secção do intestino, limitando o piloro, na parte terminal, onde o alimento entra em contato com a bile e o suco bilipancreático (Surve et al., 2017).

O Bypass gástrico em Y de Roux (ex: gastroplasia em Y de Roux ou Fobi-Capella), realizada no estômago e na parte distal do intestino delgado sendo ele duodenojejunal, é um procedimento que interfere na quantidade de ingestão alimentar e na absorção dos nutrientes. Caracteriza-se pela redução do estômago através de grampeamento, com a formação de uma bolsa gástrica, em que ocorre o desvio do duodeno, gerando a anastomose gastrojejunal (formação de um y imaginário por junção de 
dois canais), com a exclusão de parte do estômago, duodeno, e o íleo. É o procedimento considerado padrão ouro no Brasil (Peterli et al., 2018; Souza et al., 2020).

As cirurgias metabólicas do estômago e intestino delgado apresentam várias vantagens e desvantagens, conforme pode ser visto no Quadro 2:

Quadro 2: Vantagens e desvantagens das cirurgias metabólicas no estômago e intestino delgado.

\begin{tabular}{|c|c|c|}
\hline Tipo de cirurgia & Vantagens & Desvantagens \\
\hline Scopinaro & $\begin{array}{l}\text { - Perda de peso considerável; } \\
\text { - Contribui para a diminuição de } \\
\text { comorbidades. }\end{array}$ & $\begin{array}{l}\text { - Disabsortiva (contribui para o desenvolvimento de patologias } \\
\text { nutricionais); } \\
\text { - Promove diarreia (esteatorreia) caso haja o consumo de uma } \\
\text { dieta hiperlipídica; } \\
\text { - Necessita de suplementação; } \\
\text { - Irreversível; } \\
\text { - Necessidade de atentar-se ao consumo de proteínas. }\end{array}$ \\
\hline EndoBarrier & $\begin{array}{l}\text { - Realizada sem necessidade de anestesia; } \\
\text { - Rápida e prática execução; } \\
\text { - Alta no mesmo dia do procedimento; } \\
\text { - Reversível. }\end{array}$ & $\begin{array}{l}\text { - Necessário o consumo de inibidores de bomba de proteína } \\
\text { durante o tratamento; } \\
\text { - Início de uma dieta líquida antes do procedimento endobarrier e } \\
\text { pós-cirúrgico. }\end{array}$ \\
\hline Duodenal Switch & $\begin{array}{l}\text { - Contribui para a diminuição de } \\
\text { comorbidades; } \\
\text { - Perda de peso considerável. }\end{array}$ & $\begin{array}{l}\text { - Disabsortiva (contribui para o desenvolvimento de patologias } \\
\text { nutricionais); } \\
\text { - Irreversível; } \\
\text { - Promove a esteatorreia em caso do consumo de dieta } \\
\text { hiperlipídica; } \\
\text { - Promoção de refluxo gastroesofágico; } \\
\text { - Risco de desnutrição proteica. }\end{array}$ \\
\hline $\begin{array}{l}\text { Bypass em y de } \\
\text { roux } \text { ou } \\
\text { Gastroplasia em Y } \\
\text { de Roux, ou Fobi- } \\
\text { Capella }\end{array}$ & $\begin{array}{l}\text { - Perda de peso significativa e duradoura; } \\
\text { - Contribui para a redução de } \\
\text { comorbidades (hipertensão arterial e } \\
\text { diabetes mellitus); } \\
\text { - Diminuição da produção de grelina. }\end{array}$ & $\begin{array}{l}\text { - Irreversível; } \\
\text { - Disabsortiva, pois diminui a absorção de nutrientes (levando a } \\
\text { deficiências nutricionais, contribuindo para o risco nutricional); } \\
\text { - Necessária a suplementação; } \\
\text { - Promove a Síndrome de Dumping, onde o paciente apresenta } \\
\text { mal-estar ao consumir carboidratos simples. } \\
\text { - Restritivo. }\end{array}$ \\
\hline
\end{tabular}

Fonte: Autores (2021).

\subsection{Implicações pessoais das cirurgias metabólicas}

Torna-se imprescindível um estudo clínico individualizado do paciente para estabelecer os protocolos pré e póscirúrgicos (Gonçalves; Kohlsdorf; Nebra, 2020). Para que as cirurgias metabólicas sejam efetivas tornam-se necessárias alterações no estilo de vida, com a inserção de uma alimentação saudável e balanceada associada a prática de atividade física, bem como a introdução de suplementação (vitaminas do complexo B, D e ferro) em algumas cirurgias de origem mistas e disabsortivas (Andriolli, 2019; Gonçalves; Kohlsdorf; Nebra, 2020).

Algumas alterações pós-cirúrgicas são comuns, conforme destacado para os procedimentos realizados no estômago e no intestino delgado:

- O balão intragástrico pode provocar dor e desconforto, náuseas, e o refluxo gastroesofágico, úlceras gástricas e migração do balão, e em casos mais graves a perfuração do trato gastrointestinal com a pancreatite aguda e/ou morte (Coury et al., 2019). 
- Na banda gástrica ajustável pode ocorrer o deslizamento da banda, dilatação da bolsa gástrica ou erosão gástrica, e em alguns casos pode promover infecção (Chansaenroj et al., 2017; Kowalewski, 2017).

- No sleeve gástrico pode ocorrer fístula anastomótica na linha do grampeamento, após 90 dias da cirurgia, com vômitos pós-prandiais (Chung et al., 2018; Marra et al., 2021).

- Na Gastroplastia endoscópica pode promover náuseas e vômitos, em casos graves pode ocorrer o sangramento gastrointestinal e fluido perigástrico (Glass et al., 2019).

- No Scopinaro pode promover uma baixa absorção de vitaminas lipossolúveis e do complexo B, e minerais como cálcio, e ferro (Zilberstein et al., 2011).

- No EndoBarrier pode ocorrer dores abdominais e náuseas, sangramento gastrointestinal, migração do dispositivo, e em situações raras a colestase e a pancreatite (Ruban, 2018).

- No Duodenal Switch há desnutrição proteica e deficiências de vitaminas e minerais. Suas complicações se subdividem em precoces (ex: vazamento anastomótico e hemorragia) e tardias. Deve ocorrer a suplementação póscirúrgica de vitaminas lipossolúveis e do complexo B (principalmente B9 e B12), e de minerais como cálcio e ferro (Conner et al., 2021).

- No bypass gástrico em Y Roux pode ocorrer complicações no pós-operatório precoce (ex: deiscência da sutura, fístulas, esteanose, infecções e ou hemorragias), e no pós-operatório tardio (ex: hérnia interna, obstrução intestinal e/ou tromboembolismo pulmonar), e com efeitos colaterais tem-se flatulência, diarreia, doenças osteometabólicas e/ou deficiências nutricionais (Marra et al., 2021; Moura et al., 2021). Neste procedimento ocorre a má absorção vitaminas (B9, B12 e D), e ferro (Rodrigues, 2020).

- A síndrome de dumping é caracterizada por desconforto abdominal, náuseas, vômitos e complicações vasomotores como diarreias e sudorese após a ingestão de doces e alimentos gordurosos. A diminuição da digestão mecânica e da secreção de ácidos afeta diretamente a absorção de ferro, vitaminas (B12, e D), e mediante a exclusão do duodeno prejudica a absorção de minerais (cálcio, magnésio e ferro) (Nassif, 2014; Marra et al., 2021; Moura et al., 2021).

\section{Conclusão}

A obesidade é uma sindemia, cujas cirurgias metabólicas contribuem para a perda de peso de indivíduos que não foram bem-sucedidos nos demais tratamentos. Independentemente do tipo de procedimento realizado, se não houver mudanças do estilo de vida com a utilização de uma alimentação saudável, o tratamento tende a apresentar ineficácia. As cirurgias irreversíveis e disabsortivas demandam cuidados mais intensos, e por isso se faz necessário cuidados multiprofissionais e transdisciplinar, bem como a suplementação com algumas vitaminas e minerais.

A cirurgia bariátrica pode ser feita por diferentes técnicas, e cada uma apresenta particularidades e efeitos colaterais. Ambas promovem a redução de peso, contribuindo para a problemática obesidade, entretanto o indivíduo que for submetido ao procedimento, deve ser acompanhado por uma equipe multiprofissional para diminuir as complicações precoces e tardias.

Torna-se importante a realização de estudos clínicos com os pacientes submetidos à cirurgia bariátrica para que seus resultados possam ser avaliados pela equipe que irá acompanhar tal paciente, bem como gerar subsídios para a elaboração de outras revisões que poderão contribuir para a prevenção e/ou tratamento da obesidade mórbida cujo procedimento cirúrgico se faz necessário como método terapêutico para melhorar seu bem-estar e preservar sua vida. 


\section{Referências}

Almeida, L. B., \& Uhlmann, L. A. C. (2021). O uso de sibutramina para emagrecimento: uma revisão integrativa sobre os riscos e benefícios do uso desse fármaco. Pubsaúde, 1, 1-7. https://dx.doi.org/10.31533/pubsaude6.a188.

Andrade, T. B., Andrade, B. A., Jesus, J. H., \& Silva, J. N. (2019). O farmacêutico frente aos riscos do uso de inibidores de apetite: A sibutramina. Revista Científica Faema, 10, 81-92. https://doi.org/10.31072/rcf.v10iedesp.788.

Andriolli, C., Kuntz, M. G. F., Meurer, V., \& Gonçalves, A. N. (2019). Avaliação da redução de excesso de peso e de carências nutricionais em pacientes pré e pós-cirurgia bariátrica. Revista Brasileira de Obesidade, Nutrição e Emagrecimento, 11, 738-747.

Agência Nacional de Vigilância Sanitária. ANVISA. (2018). Sibutramina e remédios para emagrecer. <https://www.gov.br/anvisa/pt-br/assuntos/noticiasanvisa/2018/sibutramina-e-remedios-para-emagrecer-entenda>.

Araújo, F. K., Mourão, G. M. J., Costa, M. C. B., Alberto, N. S. M. C., Pereira, T. G., \& Ramos, C. V. (2019). Atenção nutricional para obesidade em unidades básica de saúde. Revista Brasileira de Obesidade, Nutrição e Emagrecimento, 13, 385-393.

Associação Brasileira para o Estudo da Obesidade e da Síndrome Metabólica. ABESO. (2016). Obesidade e síndrome metabólica. 4. ed. <https://abeso.org.br/wp-content/uploads/2019/12/Diretrizes-Download-Diretrizes-Brasileiras-de-Obesidade-2016.pdf>.

Associação Brasileira para o Estudo da Obesidade e da Síndrome Metabólica. ABESO. (2009). Crescem investimentos e números de cirurgias bariátricas. $<$ https://abeso.org.br/crescem-investimentos-e-numero-de-cirurgias-bariatricas/>.

Barakat, B., \& Almeida, M. E. F. (2021). Biochemical and immunological changes in obesity. Archives of Biochemistry and Biophysics, 708. https://doi.org/10.1016/j.abb.2021.108951.

Barroso, T. A., Marins, L. B., Alves, R., Gonçalves, A. C. S., Barroso, S. G., \& Rocha, G. S. (2017). Associação entre a obesidade e a incidência de doenças e fatores de risco cardiovascular. International Journal of Cardiovascular Sciences, 30, 416-424. https://doi.org/10.5935/2359-4802.20170073.

Braga, J. G. R., Concon, M. M., Lima, A. P., Callejas, G. H., Macedo, A. C., Cândido, E. C. et al. (2021). Revisional surgery in severe nutritional complications after bariatric surgical procedures: report of four cases from a single institution and review of the literature. Revista do Colégio Brasileiro de Cirurgiões, 48, 1-8. https://doi.org/10.1590/0100-6991e-20202666.

Brasil. Agência Nacional de Saúde Suplementar. (2021). Dia Mundial da Obesidade: ANS alerta para importância da coordenação do cuidado e da prevenção - ANS - Agência Nacional de Saúde Suplementar, 2021. <https://www.ans.gov.br/aans/noticias-ans/qualidade-da-saude/6219-dia-mundial-daobesidade-ans-alerta-para-importancia-da-coordenacao-do-cuidado-e-da-prevencao>.

Brasil. Conselho Federal de Medicina. CFM. $\quad$ (2017). Resolução $\quad n^{\circ} \quad$ 1.766/2005 <https://sistemas.cfm.org.br/normas/visualizar/resolucoes/BR/2005/1766>.

Brasil. Sociedade Brasileira de Cirurgia Bariátrica e Metabólica. (2019). Cirurgia para Diabetes pelo SUS já pode ser feita em Brasília. Disponível em: https://www.sbcbm.org.br/cirurgia-metabolica-diabetes-brasilia/. Acesso em: 07 fev. 2022.

Brasil. Sociedade Brasileira de Cirurgia Bariátrica e Metabólica. (2021). Nota oficial sobre a vacinação contra COVID-19 em pacientes bariátricos, 2021. https://www.sbcbm.org.br/nota-oficial-sobre-vacinacao-contra-covid-19-em-pacientes-bariatricos/.

Brasil. COJUR Brasil. Ofício CFM no 1756/2020. [2020]. 〈https://portal.cfm.org.br/images/PDF/2020_oficio_telemedicina.pdf>.

$\begin{array}{lllll}\text { Brasil. Diário } & \text { Oficial } & \text { da } & \text { União. } & \text { (2015). }\end{array}$ <https://www.in.gov.br/web/guest/materia//asset_publisher/Kujrw0TZC2Mb/content/id/22175085/do1-2016-01-13-resolucao-n-2-131-de-12-de-novembro-de2015-22174970>.

BRASIL. Ministério da Saúde. Secretaria de Atenção à Saúde. Departamento de Atenção Básica. Orientações para a coleta e análise de dados antropométricos em serviços de saúde: Norma Técnica do Sistema de Vigilância Alimentar e Nutricional. SISVAN / Ministério da Saúde, Secretaria de Atenção à Saúde, Departamento de Atenção Básica. Brasília: Ministério da Saúde, v. 76, p. 1-72, 2011.

Brasil. Vigilância de Fatores de Riscos e Proteção para Doenças Crônicas por Inquérito Telefônico. VIGITEL. (2019). Prevalência de obesidade no Brasil. <https://abeso.org.br/wpcontent/uploads/2021/07/vigitel_brasil_2019_vigilancia_fatores_risco-1-2.pdf>.

Carvalho, D. V., Gallão, M. I., \& Brito, E. S. D. E. (2020). Obesity and dietary fiber: emphasizing cashew fiber. Brazilian Journal of Development, 6, 4347443488. https://doi.org/10.34117/bjdv6n7-096.

Castilho, M. M., Westphal, G., Thon, R. A., Pereira, I. A. S., Martins, F. M., Amaral, M. F., et al. (2021). Efeitos de um programa multiprofissional de tratamento da obesidade no ambiente aquático em adultos com obesidade severa. Research, Society and Development, 10, 1-10. 10.33448/rsd-v10i1.11636.

Chansaenroj, P., Lee, W. J., Chen, S. C., Chein, J. C., \& Ser, K. H. (2017). Revision procedures after failed adjustable gastric banding: comparison of efficacy and safety. Obesity Surgery, 27, 2861-2867. DOI: 10.1007/s11695-017-2716-4.

Chung, A. Y., Thompson, R., Oyerbv, D. W., Duke, M. C., \& Farrell, T. M. (2018). Sleeve gastrectomy: surgical tips. Journal of Laparoendoscopic \& Advanced Surgical Techniques, 28, 930-937. https://doi.org/10.1089/lap.2018.0392.

Christinelli, H. C. B., Souza, J. M. S., Costa, M. A. R., Teston, E. F., Borim, M. L. C., \& Fernandes, C. A. M. Eficácia de um programa de reeducação alimentar e prática de exercício físico na obesidade. Revista Gaúcha de Enfermagem, v. 41, 2020. https://doi.org/10.1590/1983-1447.2020.20190213.

Costa, G. C., Machado, G. S. Paiva, L. V., \& Bertevello, P. L. (2020). Cirurgia bariátrica - Um tratamento para todos, a cirurgia de cada um. São Paulo: Editora Senac. 
Coury, B. F., Marques, A. C. A. S., Neto Fernandes, F. F., Semensato, J., Alves, K. N., \& Loureiro, M. C. (2019). Uma opção cirúrgica para o tratamento da migração de balão intragástrico gerando obstrução intestinal: relato de caso. Brazilian Journal of Health Review, 2, 5439-5444. 10.34119/bjhrv2n6-045.

Conner, J., \& Nottingham, J. M. (2021). Biliopancreatic diversion with duodenal switch. StatPearls, v.1, n. 25, p. 1-25, 2021.

Cuevas-Sierra, A., Lopez, O. R., Boj, J. R., Milagro, F., \& Martinez, J. A. (2019). Diet, gut microbiota, and obesity: Links with host genetics and epigenetics and potential applications. Advances in Nutrition, 10, 17-30. https://doi.org/10.1093/advances/nmy078.

De La Iglesia, R., Lorio-Kohen, V., Zulet, M. A., Martinez, J. A., Reglero, G., Molina, A. R. et al. (2016). Dietary strategies implicated in the prevention and treatment of metabolic syndrome. International Journal of Molecular Sciences, 17, 1877-1898. https://doi.org/10.3390/ijms17111877.

Dias, P. C., Henriques, P., Anjos, L. A., \& Burlandy, L. Obesidade e políticas: concepção e estratégias adotadas pelo governo brasileiro. Cadernos de Saúde Pública, v. 33, p. 1-7, 2017. https://doi.org/10.1590/0102-311X00006016.

Fortes, R. C., Sá, D. A. R., Rocha, F. M., \& Borges, W. (2021). Efeitos clínicos e nutricionais da cirurgia metabólica para indivíduos com diabetes mellitus tipo 2: políticas públicas e direitos dos usuários do Sistema Único de Saúde (SUS) no Distrito Federal. Brazilian Journal of Development, 7, $77430-77447$. $10.34117 / \mathrm{bjdv} 7 \mathrm{n} 8-114$.

Florido, L. M., Mulaski, L. F. S., Hespanhol, M. B., Mendonça, R. C. S., Santos, T. C., Silva, W. A. S. et al. (2019). Combate à obesidade: estratégias comportamentais e alimentares. Cadernos da Medicina - UNIFESO, 2, 80-89.

Gallagher, M. L. (2012). Os nutrientes e seu metabolismo. In: Mahan, L. K., Escott-Stump, S., \& Raymond, J. L. Krause alimentos, nutrição e dietoterapia. 13. ed. Rio de Janeiro: Elsevier. Cap. 3, 99-294.

Glass, J., Chaudhry, A., Zeeshan, M. S., \& Ramzan, Z. (2019). New Era: Endoscopic treatment options in obesity-a paradigm shift. World Journal of Gastroenterology, 25, 4567-4579. DOI:10.3748/wjg.v25.i32.4567.

Gonçalves, S., Kohlsdorf, M. J. G., \& Nebra, A. R. P. (2020). Adesão ao pós-operatório em cirurgia bariátrica: análise sistemática da literatura brasileira. PsicolArgum, 1, 626-646. doi: http://dx.doi.org/10.7213/psicolargum.38.102.AO02.

Kowalewski, P. K., Olszewski, R., Kwiatkowsk, A., Świderek, N. G., Cichoń, K., \& Paśnik, K. (2017). Life with a gastric band Long-term outcomes of laparoscopic adjustable gastric banding-a retrospective study. Obesity Surgery, 27, 1250-1253. 10.1007/s11695-016-2435-2.

Lima, J. A. O., Oliveira, F. M., \& Pinheiro, W. B. (2020). Diet therapy and the importance of food re-education as a health promotion for obese individuals: a literature review. Brazilian Journal of Health Review, 3, 14036-14043. 10.34119/bjhrv3n5-212.

Lubans, D., Richards, J., Hillman, C., Faulkner, G., Beauchamp, M., Nilsson, M. et al. (2016). Physical activity for cognitive and mental health in youth: A systematic review of mechanisms. Pediatrics, 138, 1-15. https://doi.org/10.1542/peds.2016-1642.

Marques, D. O., \& Quintilio, M. S. V. (2021). Farmacologia e riscos das drogas para emagrecer. Revista Coleta Científica, 1, 38-49. https://doi.org/10.5281/zenodo.5093482.

Marra, L. J., Vasconcelos, A. S., Fernandes, A. C. C., Mota, C. M., Leitão, E. V. A., Jesus, L. S. et al. (2021). Gastrectomia vertical e cirurgia de Bypass Gástrico em Y de Roux: complicações cirúrgicas e metabólicas tardias. Revista Eletrônica Acervo Científico, $29,1-7$. https://doi.org/10.25248/reac.e8127.2021.

Martínez, P. P., Mikhailidis, D. P., Athyros, V. G., Bullo, M., Couture, P., Covas, M. L. et al. (2017). Lifestyle recommendations for the prevention and management of metabolic syndrome: an international panel recommendation. Nutrition Reviews, 75, 307-326. https://doi.org/10.1093/nutrit/nux014.

Moura, G. V., Sousa, M. C., \& Lima, C. H. R. (2021). Pós-operatório de cirurgia bariátrica - uma revisão integrativa da literatura. RECIMA- Revista Científica Multidisciplinar, 2, 1-4. https://doi.org/10.47820/recima21.v2i4.26.

Murai, I. H. (2021). Nutrição e exercício na obesidade. São Paulo: Editora Senac.

Nassif, P. A. N., Malafaia, O., Ribas-Filho, J. M., Czezko, N. G., Garcia, R. F., \& Ariede, B. L. (2014). Gastrectomia vertical e bypass gástrico em Roux-em-Y induzem doença do refluxo gastroesofágico pós-operatório. ABCD. Arquivos Brasileiros de Cirurgia Digestiva, 27, 63-68. https://doi.org/10.1590/S01026720201400 S100016.

Neis, C., \& Bernardes, S. (2018). Influência do bypass gástrico em Y de Roux nos parâmetros da síndrome metabólica: uma revisão integrativa. Revista Brasileira de Obesidade, Nutrição e Emagrecimento, 12, 563-570.

Novelle, M. G., \& Diéguez, C. (2018). Food addiction and binge eating: lessons learned from animal models. Nutrients, 10, 71-95. https://doi.org/10.3390/nu10010071.

Oliveira, C. B. C., Brito, L. A., Freitas, M. A., Souza, M. P. A., Rêgo, J. M. C., \& Machado, R. J. A. (2020). Obesidade: Inflamação e compostos bioativos. Journal of Health \& Biological Sciences, 8, 1-5. http://dx.doi.org/10.12662/2317-3076jhbs.v8i1.2785.p1-5.2020.

Peterli, R., Wölnerhanssen, B. K., Peters, T., Vetter, D., Kroll, D., Borbély, Y. et al. (2018). Effect of laparoscopic sleeve gastrectomy vs laparoscopic rouxen-y gastric bypass on weight loss in patients with morbid obesity. JAMA, 319, 255-265. DOI: 10.1001/jama.2017.20897.

Prudencio, S., \& Ferreira, C. A. (2020). Departamento de Informática do SUS - DATASUS: a gestão de dados de saúde no Brasil e sua contribuição para a inclusão digital. Biblios: Revista Electrónica de Bibliotecología, Archivología y Museología, 78, 35-43. https://doi.org/10.34119/bjhrv3n6-168.

Rigoni, A. C. C., Nunes, F.G.B., \& Fonseca, K. M. (2017). O culto ao corpo e suas formas de propagação na rede social Facebook: implicações para a Educação Física escolar. Motrivivência, 29, 126-143. https://doi.org/10.5007/2175-8042.2017v29nespp126. 
Research, Society and Development, v. 11, n. 3, e13711326129, 2022

(CC BY 4.0) | ISSN 2525-3409 | DOI: http://dx.doi.org/10.33448/rsd-v11i3.26129

Rodrigues, R. C. B., Figueiredo, D. M., Mesquita, A. C., Alkimin, E. R., Peixoto, F. H. S., Leite, J. V. P. et al. (2020). Cirurgia bariátrica por bypass gástrico em Y de Roux: abordagem da técnica e de possíveis complicações tardias no pós-operatório. Revista Eletrônica Acervo Científico, 16. https://doi.org/10.25248/reac.e4979.2020.

Rossini, A. C. P., Salvador, C., Bisaio, G. T. C., Castrucci, L. S., Messenberg, M. C. C., Garrido, T. P. et al. (2021). O papel do exercício físico no tratamento da obesidade: uma revisão bibliográfica. Revista Higei@ - Revista Científica de Saúde, 2, 1-5.

Ruban, A., Ashrafian, H., \& Teare, J. P. (2018). The EndoBarrier: Duodenal-jejunal bypass liner for diabetes and weight loss. Gastroenterology Research and Practice. DOI: 10.1155 / 2018/7823182.

Santos, M. R., \& Souza, J. B. (2020). Comida como afeto, conforto e refúgio: entendendo o ato de comer em tempos de pandemia. Revista de Alimentos e Cultura das Américas - RACA, 2, 135-150. DOI: 10.35953/raca.v2i2.42.

Silva, B. C. S., Santos, S. V., \& Almeida, M. E. F. (2021). Hortas domésticas: uma fonte de saúde dentro de casa. Research, Society and Development, 10, 111. http://dx.doi.org/10.33448/rsd-v10i11.19683.

Souza, N. M. M., Santos, A. C. O., Cruz, F. S., Guimãraes, H., Silva, L. M. L., Lima, D. S. C. et al. (2020). Impacto nutricional da cirurgia bariátrica: estudo comparativo do Bypass gástrico em Y de Roux e do Sleeve entre pacientes dos sistemas público e privado de saúde. Revista do Colégio Brasileiro de Cirurgiōes, 47, 1-11. https://doi.org/10.1590/0100-6991e-20202404.

Surve, A., Zaveri, H., Cottam, D., Belnap, L., Cottam, A., \& Cottam, S. (2017). A retrospective comparison of biliopancreatic diversion with duodenal switch with single anastomosis duodenal switch (SIPS-stomach intestinal pylorus sparing surgery) at a single institution with two year follow-up. Surgery for Obesity and Related Diseases, 13, 415-422. https://doi.org/10.1016/j.soard.2016.11.020.

Swinburn, B. A., Kraak, V. I., Allender, S., Atkins, V. J., Baker, P. I., Bogard, J. R., et al. (2019). The global Syndemic of obesity, undernutrition, and climate change: The Lancet Commission Report. The Lancet, 393, 791-846. 10.1016/S0140-6736(18)32822-8

Zieba, D. A., Biernat, W., Szczesna, M., Kirsz, K., \& Misztal, L. (2019). Hypothalamic-pituitary and adipose tissue responses to the effect of resistin in sheep: The integration of leptin and resistin signaling involving a suppressor of cytokine signaling 3 and the long form of the leptin receptor. Nutrients, 11, 21802193. https://doi.org/10.3390/nu11092180.

Zilberstein, B., Santos, M. A., \& Carvalho, M. H. (2019). Análise crítica das técnicas de tratamento cirúrgico da obesidade mórbida. ABCD. Arquivos Brasileiros de Cirurgia Digestiva, 32, 1-4. https://doi.org/10.1590/0102-672020190001e1450.

Zilberstein, B., Ferreira, J. A., Carvalho, M. H., Bussons, C. Silveira-Filho, A. S., Joaquim, H. et al. (2011). Operação de Scopinaro modificado na falha da banda gástrica. ABCD. Arquivos Brasileiros de Cirurgia Digestiva, 24, 136-139. 